\title{
Cortical bone facet spacers for cervical spine decompression: effects on intervertebral kinetics and foraminal area
}

\author{
Christopher M. Maulucci, MD, ${ }^{1}$ Charles A. Sansur, MD, MHSc, ${ }^{1}$ Vaneet Singh, MS, ${ }^{2}$ \\ Alexandra Cholewczynski, BS, ${ }^{3}$ Snehal S. Shetye, PhD, ${ }^{3}$ Kirk McGilvray, PhD, ${ }^{3}$ and \\ Christian M. Puttlitz, PhD³
}

${ }^{1}$ Department of Neurosurgery, University of Maryland School of Medicine, Baltimore, Maryland; ${ }^{2}$ Medtronic Spinal and Biologics, Memphis, Tennessee; and ${ }^{3}$ Department of Mechanical Engineering and School of Biomedical Engineering, Colorado State University, Fort Collins, Colorado

OBJECTIVE Nerve root decompression to relieve pain and radiculopathy remains one of the main goals of fusionpromoting procedures in the subaxial cervical spine. The use of allograft facet spacers has been suggested as a potential alternative for performing foraminotomies to increase the space available for the cervical nerve roots while providing segmental stiffening. Therefore, the goal of this cadaveric biomechanical study was to determine the acute changes in kinetics and foraminal area after the insertion of cortical bone facet spacers into the subaxial cervical spine.

METHODS Allograft spacers ( $2 \mathrm{~mm}$ in height) were placed bilaterally into cadaveric cervical spine specimens (C2-T1, age of donors $57.5 \pm 9.5$ years, $n=7$ ) at $1(C 4-5)$ and $3(C 3-6)$ levels with and without laminectomies and posterior lateral mass screw fixation. Standard stereophotogrammetry under pure moment loading was used to assess spinal kinetics. In addition, the authors performed 3D principal component analysis of CT scans to determine changes in foraminal cross-sectional area (FCSA) available for the spinal nerve roots.

RESULTS Generally, the introduction of 2-mm-height facet spacers to the cervical spine produced mild, statistically insignificant reductions in motion with particular exceptions at the levels of implantation. No significant adjacent-level motion effects in any bending plane were observed. The addition of the posterior instrumentation (PI) to the intact spines resulted in statistically significant reductions in motion at all cervical levels and bending planes. The same kinetic results were obtained when PI was added to spines that also had facet spacers at 3 levels and spines that had been destabilized by en bloc laminectomy. The addition of 2-mm facet spacers at C3-4, C4-5, and C5-6 did produce statistically significant increases in FCSA at those levels.

CONCLUSIONS The addition of allograft cervical facet spacers should be considered a potential option to accomplish indirect foraminal decompression as measured in this cadaveric biomechanical study. However, 2-mm spacers without supplemental instrumentation do not provide significantly increased spinal segmental stability.

http://thejns.org/doi/abs/10.3171/2015.4.SPINE14845

KEY WORDS facet spacers; cervical spine; biomechanics; foraminal area

$\mathrm{P}$ OSTERIOR approaches are often preferable to anterior surgeries when treating multilevel disease of the cervical spine because the anterior approach increases the risk for dysphagia and dysphonia. ${ }^{4}$ Many posterior strategies involve extensive nerve root decompression with foraminotomies, which often require direct manipulation of the nerve root and may result in significant morbidities such as either transient or permanent palsies. ${ }^{3}$ Furthermore, foraminotomies require resection of a signif- icant amount of bone, reducing the available area for screw purchase should an instrumentation be placed. Thus, there is a critical need to develop novel posterior approach techniques that may not involve the use of foraminotomies and still accomplish the goal of neural decompression.

Previous studies have shown that distraction during anterior cervical interbody fusion procedures may play an important role in achieving functional recovery through the indirect decompression of the neural foramina and

ABBREVIATIONS 1FS = 1-level facet spacer; 3FS = 3-level facet spacer; FCSA = foraminal cross-sectional area; LAM = en bloc laminectomy; PI = posterior instrumentation; $\mathrm{ROM}=$ range of motion.

SUBMITTED August 15, 2014. ACCEPTED April 10, 2015.

INCLUDE WHEN CITING Published online September 25, 2015; DOI: 10.3171/2015.4.SPINE14845. 
spinal canal. ${ }^{1,2}$ More recently, the efficacy of cervical distraction via facet joint separation during posterior approaches has been reported. ${ }^{69}$ Goel et al. pioneered this technique by inserting metallic facet spacers to increase stability in the subaxial cervical spine, ${ }^{7}$ as well as at the atlantoaxial joint. ${ }^{5,11}$ The authors of a preliminary cadaveric study analyzing sagittal plane image data have reported that the use of subaxial machined allograft cervical facet spacers can achieve indirect decompression of the neural foramen..$^{13}$ Specifically, Tan et al. reported an average increase of $18.4 \%$ in foraminal area after bone spacers were introduced into the facet joint. ${ }^{13}$ These data provide strong support for using facet spacers to indirectly decompress the spinal nerve roots.

Although the aforementioned studies have provided valuable information about the possible efficacy of using facet spacers in the cervical spine, some important and clinically relevant issues remain unanswered and warrant further investigation. Tan et al. ${ }^{13}$ used facet shims that ranged in height from 2 to $4 \mathrm{~mm}$ (according to the size deemed appropriate for each spine specimen), but did not report specific correlations between spacer size and foraminal area increases. Therefore, it is not known whether there is a lower bound on spacer size that produces a clinically meaningful decompression (i.e., increases in foraminal area). The smallest spacer that is available for clinical use is $2 \mathrm{~mm}$ in height. This motivated our group to investigate if the introduction of these 2 -mm spacers would inherently lead to substantial increases in foraminal area and spinal stability.

In addition, given the changes in area that occur as the nerve root courses through the foramen, a more exacting study is needed of the minimum cross-sectional area available for the exiting nerve root to determine if the inclusion of facet spacers to posterior constructs will produce significant decompression. Last, it has been suggested that the introduction of the facet spacers may provide additional spinal stability via a segmental stiffening effect ${ }^{13}$ however, this increased stability has not been explicitly demonstrated. Therefore, the goal of this cadaveric biomechanical study was to determine the acute kinetic and foraminal area alterations, if any, after en bloc laminectomy (LAM) and after the insertion of cortical bone facet spacers into the subaxial cervical spine. These parameters were evaluated with and without the application of posterior instrumentation (PI).

\section{Methods \\ Experimental Groups}

To develop an experimental baseline, the intact spine specimens were first evaluated with mechanical testing and foraminal area protocols as described below. A total of 5 other experimental variants were then evaluated with each specimen. The treatment designations were based on whether the construct included PI, 1-level facet spacers (1FS), 3-level facet spacers (3FS), or LAM as follows: the PI treatment consisted of a 3-level (C3-6) PI with lateral mass screws and bilateral rods (Vertex Select Reconstruction System; Medtronic Spinal and Biologics); the 1FS treatment included 1-level (C4-5) bilateral facet spacers (CORNERSTONE Facet Micrograft, Medtronic Spi- nal and Biologics) without PI; the 3FS treatment included 3-level (C3-6) bilateral facet spacers without PI; the 3FS+LAM treatment consisted of LAM (at C3-6) with bilateral facet spacers placed in C3-6 with no PI; and the 3FS+LAM+PI treatment of LAM (at C3-6) with bilateral facet spacers placed in C3-6 and PI spanning C3-6.

Figure 1 shows digital images of the facet spacer and of the 6 experimental variants. All facet spacers measured $8 \mathrm{~mm}$ in width, $8 \mathrm{~mm}$ in length, and $2 \mathrm{~mm}$ in height. Surgical preparation of the samples was performed by an experienced spine surgeon.

\section{Sample Preparation and Biomechanical Analysis}

Seven fresh-frozen, human cadaveric cervical spines (comprising levels C2-T1) were used in this study (the mean age of the donors was $57.5 \pm 9.5$ years.). Care was taken to dissect extraneous soft tissue from the samples without disrupting the osseous and ligamentous architecture. The C-2 and T-1 vertebral bodies were potted in a 2-part epoxy resin that, when fully cured, allows mounting of the sample on the spine-testing equipment. The potted vertebral body of C-2 was rigidly coupled to a robotic actuator, and the potted vertebral body of $\mathrm{T}-1$ was rigidly fixed to a multiaxial force-moment-sensing transducer. The testing apparatus used an in-line torque motor and a torque load cell to apply pure moments (up to a maximum of $1.5 \mathrm{~N}-\mathrm{m}$ ) to the specimen in flexion-extension, right and left lateral bending, and right and left axial rotation. A translational X-Y table and counterweight system were used to remove offset and out-of-plane moment or shear forces and to balance the weight of the upper portion of the fixture, ensuring pure moment loading in the direction of interest.

Three noncollinear markers, forming "tracking triads," were placed at the tips of $\mathrm{K}$ wires and drilled into each vertebral body. Three high-resolution cameras (Motion Analysis) and custom-designed algorithms (LabView 8.0) were used to calculate 3D displacements (i.e., intervertebral rotations) with the principles of stereo photogrammetry. The resulting data were used to calculate the kinetic range of motion (ROM) across the spinal levels involved and at the adjacent segments for all 3 bending planes. Samples were preconditioned for 3 cycles, and data were collected on the fourth cycle at $100 \mathrm{~Hz}$. Post hoc data analysis included plotting the moment-rotation relationships, and ROMs were calculated as the absolute difference between the maximum and minimum datum points (at $1.5 \mathrm{~N}-\mathrm{m}$ ). A 1-way ANOVA was performed (Sigma Stat 3.1) to determine statistically significant $(\alpha=0.05)$ differences in ROM among treatments.

\section{CT Analysis}

Following each battery of biomechanical tests (i.e., loading in all 3 bending planes), each surgical variant (with the exception of 1FS, i.e., the 1-level bilateral cortical facet spacers in C4-5) was scanned with a clinical (176-micron in-plane resolution) CT scanner (Gemini TF Big Bore Scanner) to determine the degree of neural foramen decompression (if any) afforded by each instrumentation group. Foam supports were used to orient the samples in a repeatable manner within the CT bore and to ensure that the samples were placed in a neutral bending 


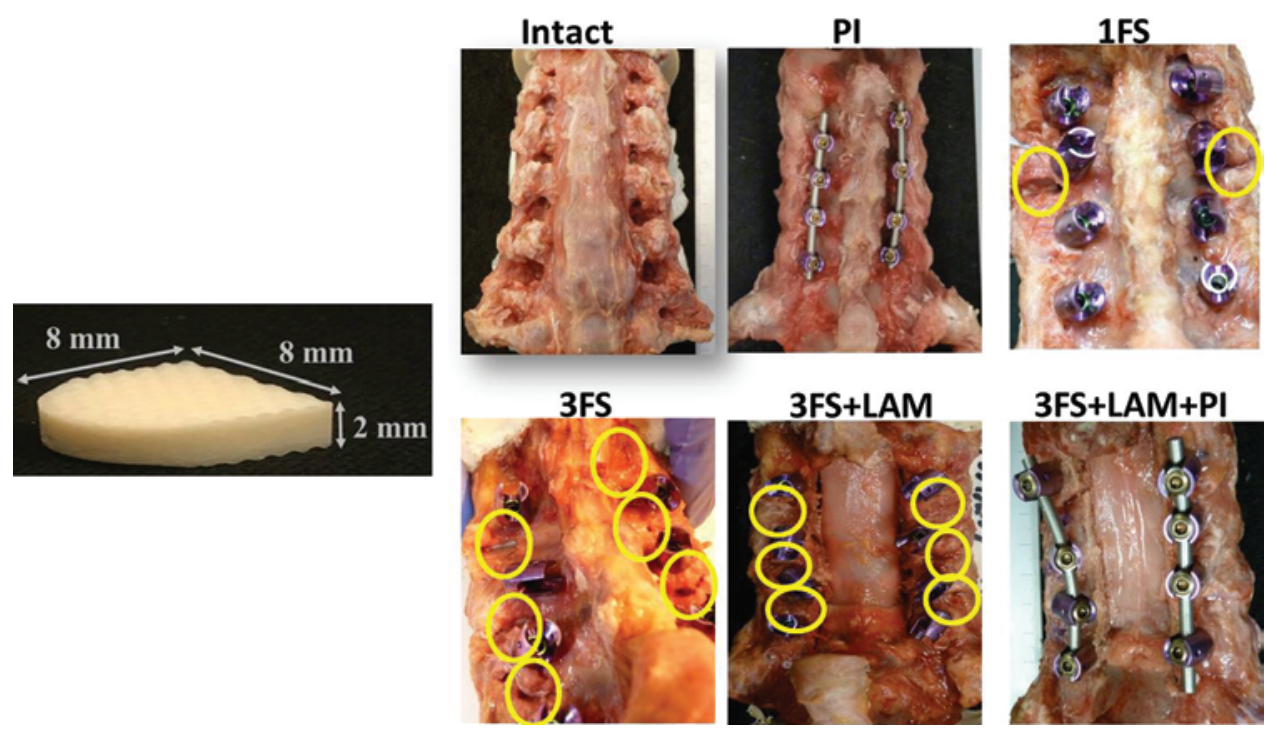

FIG. 1. Images of an $8 \times 8 \times 2-\mathrm{mm}$ facet spacer (left) and of all 6 experimental variants that were evaluated in this study (right). Each treatment variant was applied to all 7 cervical spines used in this investigation. Bilateral facet spacer placements are highlighted in yellow circles (except for the 3FS+LAM+PI treatment because the Pl occluded visualization of the facet spacers). Figure is available in color online only.

configuration before scanning. The 3D reconstructions of each sample involved a generalized bone algorithm with $0.8 \mathrm{~mm} \times 0.4 \mathrm{~mm}$ (scan thickness $\times$ increment) craniocaudal resolution embedded within a software platform for visualizing and manipulating biomedical image data (Amira; Visage Imaging, Inc.). These data manipulations allowed isolation of each cervical nerve foramen of interest (bilaterally at the 3 treated levels, i.e., at C3-4, C4-5, and C5-6) (Fig. 2).

Three anatomical landmarks that have been used in previous studies of foraminal areas ${ }^{8,10,12}$ were identified: 1) the center of the medial-lateral ridge of the inferior edge of the pedicle on the superior vertebral body, 2) the center of the medial-lateral ridge of the superior edge of the pedicle on the inferior vertebral body, and 3) the anterior medial inferior articular process of the facet on the inferior vertebral body. Using these landmarks, we created a plane that bisected the foraminal cross-sectional area (FCSA) (Fig. 2). The line set circumscribing the boundary of the FCSA was imported into Matlab (R2010b Version 7.11; MathWorks Inc.), and a custom-written code was used to calculate the FCSA. Specifically, the minimum principal components of the 3D and projected FCSAs were determined, and the area of the polygon enclosed by the FCSA boundary was calculated. The FCSA was averaged for each functional spinal unit (e.g., the C3-4 right FCSA and the C3-4 left FCSA were averaged together for the reported C3-4 FCSA measurement).

To determine intraobserver variation in the FCSA measurements, a single investigator, blinded to image-identifying information, measured a subset of images twice on 2 separate occasions, resulting in a total of 16 measurements. Intraobserver variations of these measurements were analyzed with a 1-way, repeated-measures ANOVA. The ANOVA results indicated that the differences in the median values among the redundant single-observer measurements were not large enough to exclude the possibility that these differences were due to random sampling variability $(\mathrm{p}$ value $=0.99)$.

To study interobserver variation, 2 different examiners, also blinded to image-identifying information, measured the same series of samples (16 measurements per observer). Interobserver variations of the results were also analyzed with a 1-way, repeated-measures ANOVA. The differences in the medians among the redundant measurements made by the 2 observers were again not large enough to exclude the possibility that these differences were due to random sampling variability. Paired t-tests were used to delineate differences $(\alpha=0.05)$ among the treatment groups.

\section{Results}

\section{Biomechanical Loading Analysis}

As expected, the kinetic data indicated that the addition of PI to an intact spine statistically significantly reduces motion at all cervical levels and bending planes (Figs. 3-5). The same results were obtained when PI was added to spines that also include facet spacers at 3 levels (C3$6)$ and that were destabilized via LAM (3FS+LAM+PI). These results confirmed the expected outcome, indicating that after PI is applied, it assumes the dominant mechanical stability role in the cervical spine.

Generally, the introduction of facet spacers to the cervical spine produced mild, statistically insignificant reductions in motion (with certain exceptions noted below) at the level(s) of implantation. Specifically, compared with the intact condition, placement of a 2-mm facet spacer at C4-5 (1FS) tended to reduce average motion by $2.7^{\circ}, 2.9^{\circ}$, and $3.1^{\circ}$ in flexion or extension, lateral bending, and axial rotation, respectively. No significant adjacent-level motion effects due to the 1FS treatment were observed in any bending plane at C3-4 and C5-6. Compared with motion in the intact spine, the introduction of bilateral facet spac- 


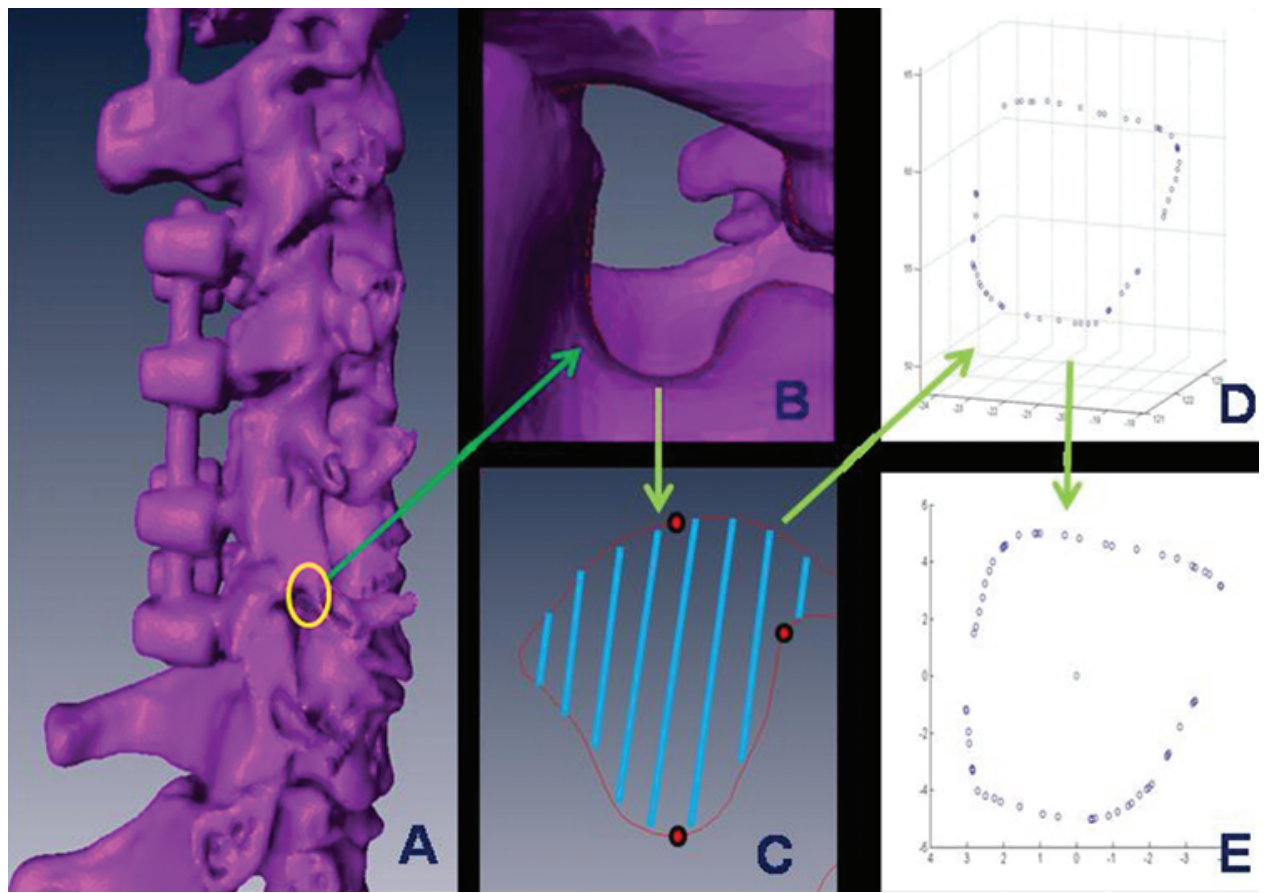

FIG. 2. A 3D reconstruction of the cervical spine CT scans was used to perform the FCSA analysis (A). Each foramen was identified and isolated for analysis of the area (B). A plane bisecting the FCSA was defined by fitting an analytical function to 3 anatomical landmark points, and an area definition was thus created (C). This area was projected in 3D space, and its minimum 3 principal components were calculated (D). Resolution of the principal components resulted in the final areal measurement (E). Figure is available in color online only.

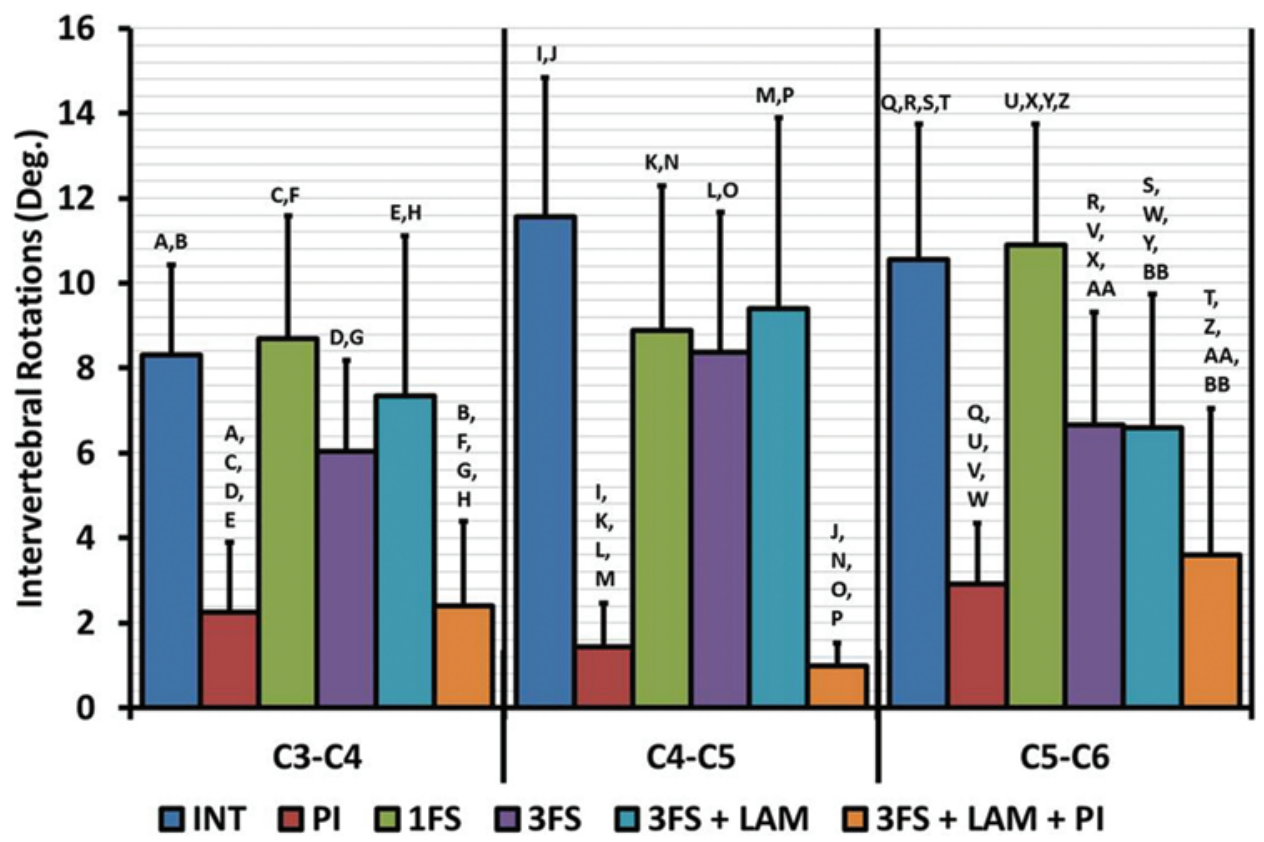

FIG. 3. Flexion-extension kinetic ROM data for cervical spine levels C3-4, C4-5, and C5-6 under flexion and extension. Data represent the mean; error bars indicate SD. Identical letters above the bars indicate statistically significant differences between treatments: $A(p<0.001), B(p<0.001), C(p<0.001), D(p=0.022), E(p=0.003), F(p<0.001), G(p=0.011), H(p=0.002), I(p$ $<0.001), J(p<0.001), K(p<0.001), L(p<0.001), M(p<0.001), N(p<0.001), O(p<0.001), P(p<0.001), Q(p<0.001), R(p=$ $0.029), S(p=0.017), T(p=0.011), U(p<0.001), V(p=0.006), W(p=0.016), X(p=0.014), Y(p=0.015), Z(p=0.002), A A(p=$ $0.026)$, and BB $(p=0.038)$. INT = intact. Figure is available in color online only. 


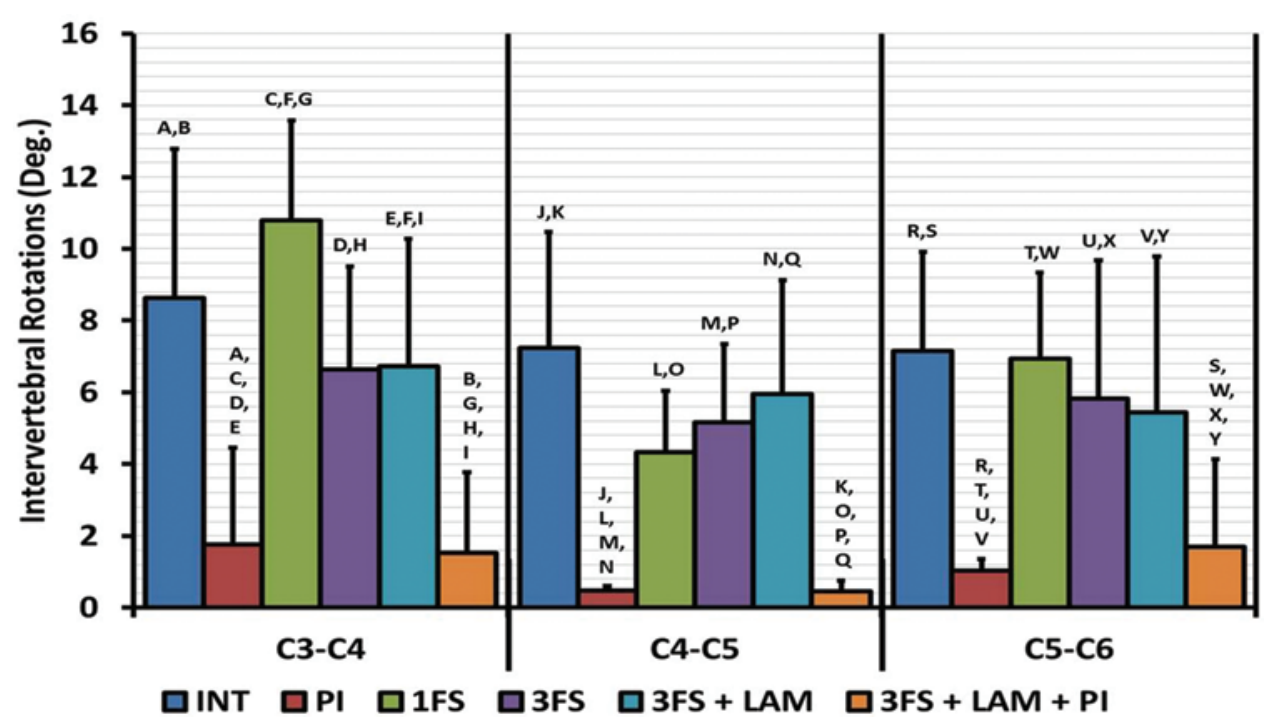

FIG. 4. Lateral bending kinetic ROM data for cervical spine levels C3-4, C4-5, and C5-6 under lateral bending. Data represent the mean; error bars indicate SD. Identical letters above the bars indicate statistically significant differences between treatments: $A(p=0.001), B(p=0.001), C(p<0.001), D(p=0.006), E(p=0.014), F(p=0.049), G(p<0.001), H(p=0.011), I(p=0.018), J(p$ $<0.001), K(p<0.001), L(p<0.001), M(p<0.001), N(p<0.001), O(p<0.001), P(p<0.001), Q(p<0.001), R(p<0.001), S(p=$ $0.002), T(p<0.001), U(p<0.001), V(p<0.001), W(p=0.007), X(p=0.011)$, and $Y(p=0.026)$. Figure is available in color online only.

ers in C3-4, C4-5, and C5-6 (3FS) also statistically insignificantly reduced motion at every level. Compared with the intact spine motion, we noted a significant reduction due to $3 \mathrm{FS}$ in $\mathrm{C} 3-4$ axial rotation motion $\left(4.4^{\circ}, \mathrm{p}=0.007\right)$ and C5-6 flexion-extension motion $\left(4.0^{\circ}, \mathrm{p}=0.029\right)$. Compared with the 3 FS treatment, the addition of LAM to the
3FS construct (3FS+LAM) had no significant effect on cervical motion in any plane at any level. Last, no statistically significant motion changes due to instrumentation in the C3-6 levels were observed in any bending plane in the adjacent functional spinal units for both the cranial (C2-3) and the caudal (C6-7 and C7-T1) levels (Table 1)

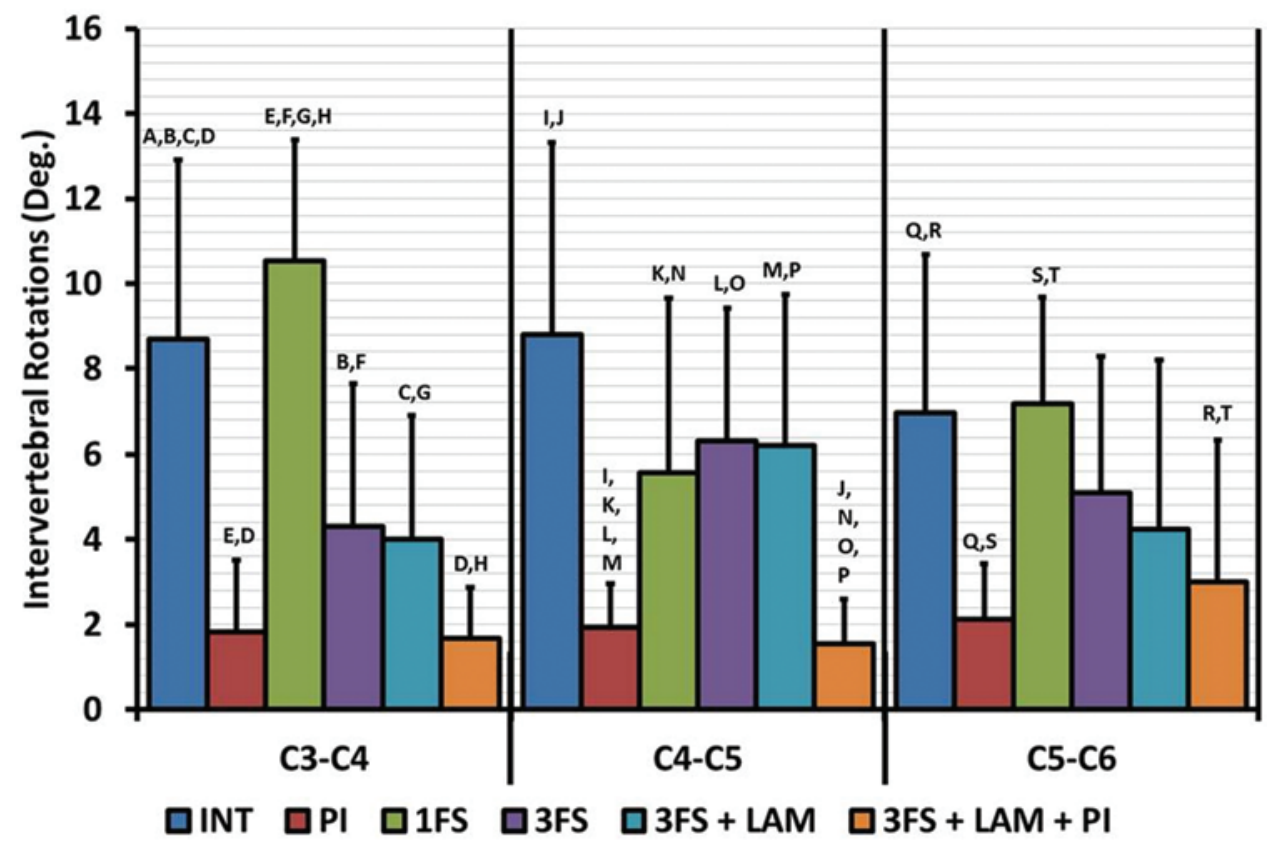

FIG. 5. Axial rotation kinetic ROM data for cervical spine levels C3-4, C4-5, and C5-6 under axial rotation. Data represent the mean; error bars indicate SD. Identical letters above the bars indicate statistically significant differences between treatments: $A$ $(p<0.001), B(p=0.007), C(p=0.012), D(p<0.00), E(p<0.001), F(p<0.001), G(p<0.001), H(p<0.001), I(p=0.001), J(p=$ $0.001), K(p=0.038), L(p=0.001), M(p=0.017), N(p=0.038), O(p=0.001), P(p=0.017), Q(p=0.002), R(p=0.008), S(p=$ $0.002)$, and $T(p=0.008)$. Figure is available in color online only. 
TABLE 1. Kinetic ROM data at the 3 adjacent cervical spine levels for the spine treatments in this study*

\begin{tabular}{|c|c|c|c|c|c|c|c|}
\hline \multirow[b]{2}{*}{ Vertebral Level/Motion } & \multicolumn{6}{|c|}{ Spine Treatment } & \multirow[b]{2}{*}{$p$ Value } \\
\hline & None (intact) & PI & 1FS & $3 \mathrm{FS}$ & $3 F S+L A M$ & $3 F S+L A M+P I$ & \\
\hline \multicolumn{8}{|l|}{$\mathrm{C} 2-3$} \\
\hline Axial rotation & $3.0(2.7)$ & $3.9(1.6)$ & $3.4(2.4)$ & $4.0(2.7)$ & $3.8(2.7)$ & $4.3(1.7)$ & 0.7 \\
\hline Flex/ext & $6.7(1.4)$ & $7.0(1.4)$ & $5.9(2.6)$ & $6.9(1.7)$ & $7.1(1.6)$ & $7.5(1.6)$ & 0.7 \\
\hline Lat bending & $8.2(2.8)$ & $9.7(3.3)$ & $10.8(4.0)$ & $9.9(2.8)$ & $9.2(4.3)$ & $9.7(3.4)$ & 0.8 \\
\hline \multicolumn{8}{|l|}{ C6-7 } \\
\hline Axial rotation & $4.7(1.7)$ & $11.2(3.1)$ & $7.1(4.7)$ & $6.4(2.6)$ & $4.4(2.7)$ & $6.6(2.5)$ & 0.5 \\
\hline Flex/ext & $10.9(3.2)$ & $10.9(3.2)$ & $11.3(2.9)$ & $9.4(2.7)$ & $11.3(3.6)$ & $14.2(5.5)$ & 0.3 \\
\hline Lat bending & $6.6(1.8)$ & $6.3(2.1)$ & $7.6(1.9)$ & $6.4(2.2)$ & $7.2(1.9)$ & $7.2(1.9)$ & 0.8 \\
\hline \multicolumn{8}{|l|}{$\mathrm{C} 7-\mathrm{T} 1$} \\
\hline Axial rotation & $4.7(2.7)$ & $9.2(1.3)$ & $7.4(4.0)$ & $4.2(1.7)$ & $3.7(2.3)$ & $4.9(2.3)$ & 0.1 \\
\hline Flex/ext & $6.1(2.9)$ & $5.8(2.5)$ & $7.0(3.5)$ & $5.8(3.7)$ & $5.9(2.6)$ & $7.4(3.8)$ & 0.9 \\
\hline Lat bending & $5.5(4.2)$ & $4.6(2.3)$ & $4.6(2.6)$ & $4.7(1.8)$ & $4.2(3.3)$ & $3.5(1.2)$ & 0.8 \\
\hline
\end{tabular}

\section{FCSA Analysis}

The data indicated that the addition of PI to an intact spine does not result in significant changes in the FCSA (Fig. 6). However, the addition of 2-mm facet spacers did produce statistically significant increases in FCSA at the implanted levels. For example, compared with the intact condition, statistically significant increases in the FCSA across the C3-4, C4-5, and C5-6 levels were observed for the 3FS $(6.4 \%, 3.3 \%$, and $5.0 \%$ increases, respectively) and $3 \mathrm{FS}+\mathrm{LAM}(6.3 \%, 3.0 \%$, and $4.7 \%$ increases, respectively) groups. Statistically significant increases $(\mathrm{p}<0.001$ for all comparisons) in the FCSA were also observed when comparing the FCSA of the groups with the facet spacers (namely, the 1FS, 3FS, and 3FS+LAM experimental variants) with that of the PI-only group. Overall, the addition of LAM (3FS+LAM) and of PI (3FS+LAM+PI) to the

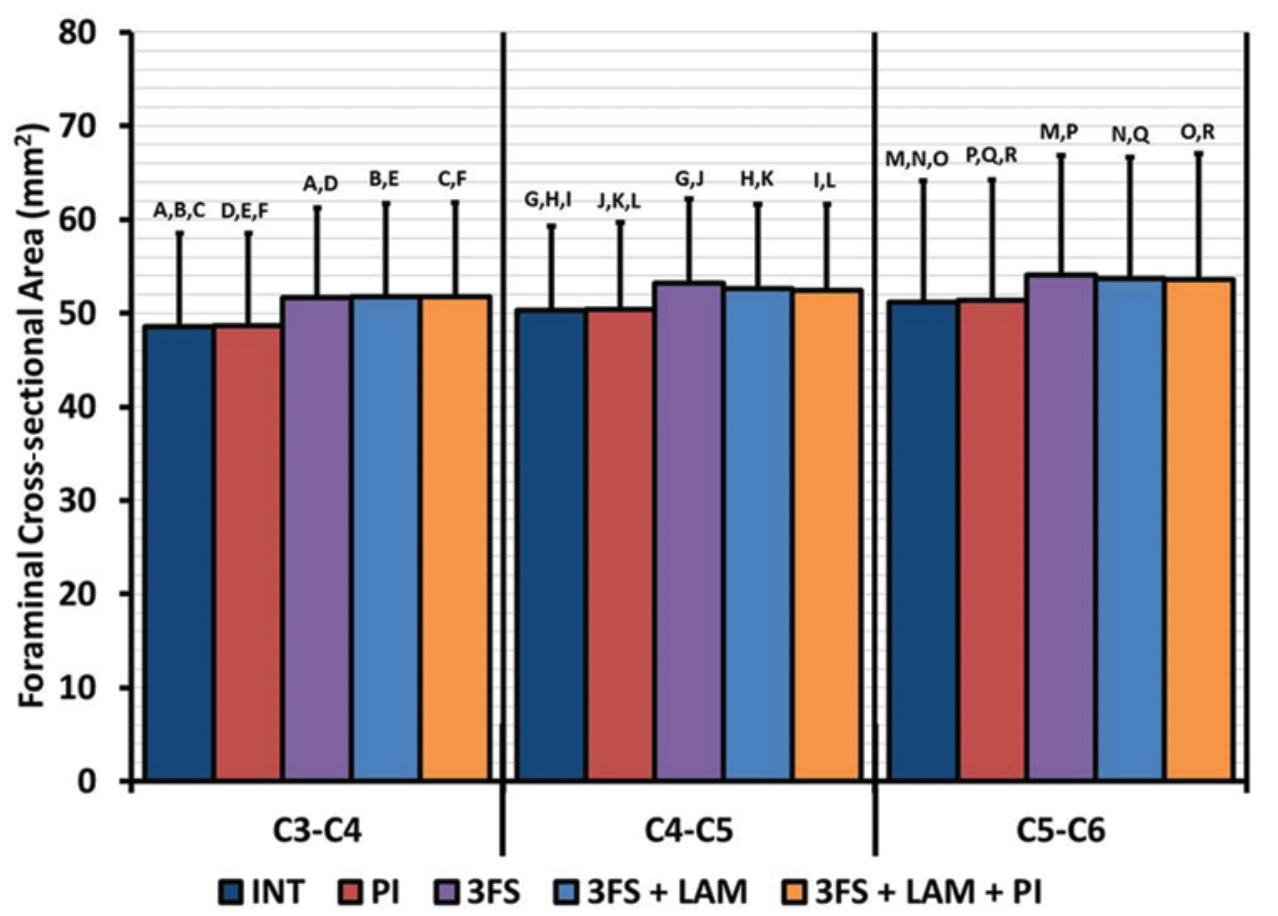

FIG. 6. FCSA measured at the cervical spine levels C3-4, C4-5, and C5-6. Data represent the mean; error bars indicate SD. Identical letters above the bars indicate statistically significant differences between treatments: $A(p<0.001), B(p<0.001), C(p$ $<0.001), D(p<0.001), E(p<0.001), F(p<0.001), G(p<0.001), H(p=<0.001), I(p<0.001), J(p<0.001), K(p<0.001), L(p<$ $0.001), M(p<0.001), N(p<0.001), O(p=0.013), P(p<0.001), Q(p<0.001)$, and $R(p<0.001)$. Figure is available in color online only. 
cervical spine with facet spacers at the 3 levels did not further increase the FCSA because the facet spacers already maintained a significantly greater FCSA than in both the intact and the PI spines.

\section{Discussion}

Posterior procedures to decompress and stabilize the cervical spine are most often performed to treat patients with cervical spondylotic myelopathy because this disease process very frequently involves stenosis at multiple levels. However, cervical radiculopathy often exists concurrently, and may represent a patient's primary reason for seeking medical attention. When foraminal stenosis coexists with central canal stenosis, both pathologies may be addressed with the posterior approach. However, cervical foraminotomies involve bone removal, which may hinder hardware purchase or provide less surface area for arthrodesis. Furthermore, the addition of foraminotomies to a procedure may increase operative time and blood loss because of the dense venous network that surrounds the cervical nerve roots. Moreover, authors of a large clinical series have recently reported that the performance of cervical foraminotomy is the main predictor of $\mathrm{C}-5$ nerve palsy in patients undergoing posterior cervical surgery. ${ }^{3}$

In theory, the insertion of an allograft facet spacer addresses the main goals of arthrodesis-promoting surgery, namely, foraminal decompression and increased surface area available for fusion. The removal of the cartilaginous articular surface (with associated bleeding subchondral bone) and the insertion of an allograft spacer into the facet joint may aid in achieving a successful fusion.

We hypothesized that the insertion of 2-mm height allograft facet spacers without supplemental segmental instrumentation would provide significant increases in segmental stiffness. However, when compared with the intact spine condition, the 1-level (1FS) and 3-level spacer (3FS) groups did not show significant reductions in ROM in all planes at all levels except for a reduction at C3-4 in axial rotation and at C5-6 in flexion-extension with 2-mm spacers. Thus, from these results we conclude that the introduction of 2-mm spacers into the facet joints does not provide a substantial stiffening effect.

Although the kinetic data indicated that smaller facet spacers as stand-alone devices do not significantly reduce acute spinal motion, the data clearly showed that placement of the 2-mm facet spacers significantly increases FCSA. The FCSA after spacer placement was, on average, $5 \%$ larger than in the intact spine. The cervical nerve roots and ganglions pass through the foramen at or below the levels of the uncovertebral and zygapophysial joint lines with the large sensory roots superior and dorsal to the small motor roots. ${ }^{14}$ The cervical intervertebral foramina are almost entirely filled with the cervical dorsal root ganglions. For these anatomical reasons, it seems reasonable to assume that cervical radiculopathy is strongly correlated with both the cross-sectional area and shape of the intervertebral foramen. ${ }^{14}$ We hypothesize that small increases in the FCSA can reduce the compression on the nerve root, and thus decrease associated acute and chronic nerve disorders. However, the amount of decompression required to completely eliminate or to partially diminish nerve root-associated radiculopathy remains unclear, and it is therefore difficult to rigorously correlate the clinical relevance of the FCSA changes reported here.

\section{Effect of Facet Spacer Height}

Given the aforementioned kinetic and FCSA results obtained with 2-mm facet spacers, we performed a set of pilot experiments to evaluate any effects of the facet spacer height on spinal stability and on the space available for the nerve roots. For these preliminary experiments, we used 2 cadaveric cervical spine sections and followed the experimental protocol as described in Methods. Each specimen was evaluated in its intact condition and then implanted with 3-mm height facet spacers at C3-4, C4-5, and C5-6 bilaterally without PI. The specimens were then kinetically evaluated and scanned with CT. The 3-mm height spacers were replaced with 4-mm height spacers and again kinetically and radiographically reevaluated.

The data shown in Table 2 support the contention that increasing the height of the facet spacers produces substantial increases in spinal stability and stiffening. For example, the kinetics of the intact spine were reduced by $85 \%$ or more in all 3 bending planes when $4-\mathrm{mm}$ bilateral spacers were introduced into the facet spaces at the 3 cervical levels. In addition, the 4-mm spacers increased the FCSA at these levels by approximately $20 \%$. These data clearly indicate the substantial stiffening effect, that is, the reduced kinetics that is associated with increasing spacer height. Similarly, the FCSA was greatly increased with the introduction of 3-mm and 4-mm spacers to the subaxial cervical facets.

TABLE 2. Percentage reductions in segmental kinetics and percentage increases in foraminal area due to the placement of 2-, 3-, and 4-mm spacers at the $\mathrm{C} 3-4, \mathrm{C} 4-5$, and C5-6 vertebral levels without $\mathrm{PI}^{*}$

\begin{tabular}{|c|c|c|c|c|}
\hline \multirow[b]{2}{*}{ Variable } & \multirow{2}{*}{$\begin{array}{c}\text { Spacer } \\
\text { Height } \\
(\mathrm{mm})\end{array}$} & \multicolumn{3}{|c|}{$\begin{array}{c}\text { Changes at Vertebral } \\
\text { Levels }(\%)\end{array}$} \\
\hline & & C3-4 & C4-5 & C5-6 \\
\hline \multicolumn{5}{|c|}{ Reductions in segmental kinetics } \\
\hline \multicolumn{5}{|c|}{ Axial rotation } \\
\hline & 2 & 50.5 & 41.2 & 26.8 \\
\hline & 3 & 87.8 & 73.0 & 87.7 \\
\hline & 4 & 94.3 & 85.8 & 93.0 \\
\hline \multicolumn{5}{|l|}{ Lat bending } \\
\hline & 2 & 23.1 & 28.7 & 18.5 \\
\hline & 3 & 67.5 & 76.9 & 44.3 \\
\hline & 4 & 99.6 & 95.5 & 95.9 \\
\hline \multicolumn{5}{|l|}{ Flexion-extension } \\
\hline & 2 & 27.5 & 27.5 & 36.9 \\
\hline & 3 & 58.3 & 57.4 & 51.5 \\
\hline & 4 & 93.5 & 95.5 & 98.5 \\
\hline \multicolumn{5}{|c|}{ Increases in foraminal area } \\
\hline & 2 & 6.5 & 4.3 & 5.8 \\
\hline & 3 & 13.0 & 15.0 & 12.2 \\
\hline & 4 & 19.5 & 20.4 & 21.2 \\
\hline
\end{tabular}

* The 2-mm spacer data represent the means from the entire study $(n=7)$, whereas the $3-\mathrm{mm}$ and $4-\mathrm{mm}$ spacer data represent the means of a pilot study $(n=2)$. 
Although based on a very small sample size, the results of the experiments with the 3-mm and 4-mm facet spacers shown in Table 2 support the hypothesis that larger spacers offer substantial acute stability to the cervical spine while providing nerve root decompression. This option may be particularly useful in elderly patients with osteoporosis, whose bone quality may therefore not be amenable to obtaining the instrumentation purchase that is required to avoid subsequent hardware loosening and pseudarthrosis.

\section{Limitations}

As with any cadaver study that seeks to mimic an in vivo environment, certain limitations must be considered. The results reported here represent the acute, immediately postoperative kinetic and foraminal area changes a surgeon might expect after including facet spacers in a cervical construct. Further cyclic experiments are required to predict the more long-term behavior of these implants. In addition, the kinetic evaluations in this study used pure moment loading to simulate the muscular action present in vivo to produce bending in all 3 planes. Although this is a well-accepted method, it certainly represents a simplification of the in vivo loading environment. Nevertheless, in spite of these in vitro representations of the in vivo milieu, the method employed in this study was robust to provide a direct comparison of different fixation-implantation scenarios.

We note that because the procedures required some tissue resection, a fully randomized experimental design was not possible. For example, it is necessary to remove the facet capsules to place the facet spacers. Therefore, the 1FS procedure was performed first, followed by the 3-level procedures (3FS, 3Fs+LAM, and 3FS+LAM+PI). Last, the main portion of the study evaluating $2-\mathrm{mm}$ spacers involved a full set of 7 spinal specimens, but the subsequent pilot study with the 3-mm and 4-mm height spacers used only 2 specimens. Therefore, even though the latter data showed dramatic increases in foraminal area and decreases in segmental kinetics with increasing spacer height, these results must be viewed within the context of a limited specimen pool and warrant further investigation.

\section{Conclusions}

In conclusion, the addition of allograft cervical facet spacers should be considered a potential option to accomplish indirect foraminal decompression. We found that facet spacers $2 \mathrm{~mm}$ in height significantly increased the FCSA. However, without supplemental instrumentation, the 2-mm spacers did not provide significantly increased spinal segmental stability. Additional investigations are required with larger (greater than $2 \mathrm{~mm}$ in height) facet spacers to 1) more rigorously characterize the kinematic changes associated with the placement of these larger implants, 2) more solidly determine whether they can be safely implanted, and 3) assess whether additional clinical benefits may be obtained with their use.

\section{References}

1. Albert TJ, Smith MD, Bressler E, Johnson LJ: An in vivo analysis of the dimensional changes of the neuroforamen after anterior cervical diskectomy and fusion: a radiologic investigation. J Spinal Disord 10:229-233, 1997
2. Bayley JC, Yoo JU, Kruger DM, Schlegel J: The role of distraction in improving the space available for the cord in cervical spondylosis. Spine (Phila Pa 1976) 20:771-775, 1995

3. Bydon M, Macki M, Kaloostian P, Sciubba DM, Wolinsky JP, Gokaslan ZL, et al: Incidence and prognostic factors of C5 palsy: a clinical study of 1,001 cases and review of the literature. Neurosurgery 74:595-605, 2014

4. Chen CJ, Saulle D, Fu KM, Smith JS, Shaffrey CI: Dysphagia following combined anterior-posterior cervical spine surgeries. J Neurosurg Spine 19:279-287, 2013

5. Goel A: Atlantoaxial joint jamming as a treatment for atlantoaxial dislocation: a preliminary report. Technical note. J Neurosurg Spine 7:90-94, 2007

6. Goel A: Facet distraction-arthrodesis technique: Can it revolutionize spinal stabilization methods? J Craniovertebr Junction Spine 2:1-2, 2011

7. Goel A, Shah A: Facetal distraction as treatment for singleand multilevel cervical spondylotic radiculopathy and myelopathy: a preliminary report. J Neurosurg Spine 14:689696, 2011

8. Ivancic PC: Cervical neural space narrowing during simulated rear crashes with anti-whiplash systems. Eur Spine J 21:879-886, 2012

9. McCormack BM, Bundoc RC, Ver MR, Ignacio JM, Berven SH, Eyster EF: Percutaneous posterior cervical fusion with the DTRAX Facet System for single-level radiculopathy: results in 60 patients. J Neurosurg Spine 18:245-254, 2013

10. Muhle C, Resnick D, Ahn JM, Südmeyer M, Heller M: In vivo changes in the neuroforaminal size at flexion-extension and axial rotation of the cervical spine in healthy persons examined using kinematic magnetic resonance imaging. Spine (Phila Pa 1976) 26:E287-E293, 2001

11. Park J, Scheer JK, Lim TJ, Deviren V, Ames CP: Biomechanical analysis of Goel technique for C1-2 fusion. J Neurosurg Spine 14:639-646, 2011

12. Takasaki H, Hall T, Jull G, Kaneko S, Iizawa T, Ikemoto Y: The influence of cervical traction, compression, and Spurling test on cervical intervertebral foramen size. Spine (Phila Pa 1976) 34:1658-1662, 2009

13. Tan LA, Gerard CS, Anderson PA, Traynelis VC: Effect of machined interfacet allograft spacers on cervical foraminal height and area. J Neurosurg Spine 20:178-182, 2014

14. Tominaga Y, Maak TG, Ivancic PC, Panjabi MM, Cunningham BW: Head-turned rear impact causing dynamic cervical intervertebral foramen narrowing: implications for ganglion and nerve root injury. J Neurosurg Spine 4:380-387, 2006

\section{Disclosure}

Institutional research support grant funding for this study was provided by Medtronic Spinal and Biologics to Colorado State University. Dr. Puttlitz is a consultant for Medtronic Spinal and Biologics; Dr. Sansur is a consultant for Medtronic and DePuy Synthes; and Mr. Singh is an employee of Medtronic.

\section{Author Contributions}

Conception and design: Puttlitz, Maulucci, Sansur, Singh. Acquisition of data: all authors. Analysis and interpretation of data: Puttlitz, Maulucci, Sansur, Cholewczynski, McGilvray. Drafting the article: Puttlitz, Maulucci, Sansur, Singh. Critically revising the article: Puttlitz, Maulucci, Sansur, Singh. Reviewed submitted version of manuscript: Puttlitz, Maulucci, Sansur, Singh, McGilvray. Approved the final version of the manuscript on behalf of all authors: Puttlitz. Statistical analysis: Puttlitz, Cholewczynski, Shetye, McGilvray. Administrative/technical/ material support: Puttlitz. Study supervision: Puttlitz.

\section{Correspondence}

Christian M. Puttlitz, Department of Mechanical Engineering, School of Biomedical Engineering, 1374 Campus Delivery, Colorado State University, Fort Collins, CO 80523-1374. email: puttlitz@engr.colostate.edu. 